\title{
Automedicación en salud bucal en indígenas Muiscas, Yanakunas y Pijaos de Colombia
}

\author{
Oral health self-medication in Muiscas, Yanakunas and Pijaos \\ indigenous populations of Colombia
}

Edson Jair Ospina Lozano (https://orcid.org/0000-0002-4040-091X) ${ }^{1}$

Yesica Daniela Liscano Pinzón (https://orcid.org/0000-0002-0534-9100) ${ }^{1}$

Sergio Iván Pachón Solano (https://orcid.org/0000-0002-4173-1980) ${ }^{1}$

${ }^{1}$ Facultad de Odontología, Universidad Nacional de Colombia. Carrera 30 45-03, Edificio 210. Bogotá Colombia.

ejospinal@unal.edu.co

\begin{abstract}
Self-medication is a social practice that corresponds to the autonomous use of therapeutic elements to address diseases. It involves a sociocultural context of the disease, and a rationality focused on therapeutic efficacy and the availability of curative supplies. Identify and analyze the process of oral health self-medication in indigenous Muiscas, Pijaos, and Yanakunas of Colombia. This is a qualitative, ethnographic study employing techniques such as documentary analysis, participant observation, field diary, and ethnographic interviews. Self-medication is frequent among these indigenous people. It prioritizes the use of pharmaceutical drugs in the Muiscas and medicinal plants in the Yanakunas. The therapeutic supplementation is evident in the Pijaos. The pharmaceutical elements focus on a matter of social progress and the herbal elements on a matter of cultural identity. In these communities, oral health self-medication is a structured and daily practice in families to address their ailments. This practice is supported by the knowledge, management, and availability of different therapeutic resources.
\end{abstract}

Key words Oral Health, Self Medication, Indigenous Population, Qualitative Research, Medicinal Plants
Resumen La automedicación es una práctica social que corresponde al uso autónomo de elementos terapéuticos para enfrentar las enfermedades. Involucra un contexto sociocultural de la enfermedad, una racionalidad centrada en la eficacia terapéutica y la disponibilidad de insumos curativos. Identificar y analizar el proceso de automedicación en salud bucal en indígenas Muiscas, Pijaos y Yanakunas de Colombia. Estudio cualitativo, con enfoque etnográfico, y uso de técnicas como análisis documental, observación participante, diario de campo y entrevistas etnográficas. La automedicación es frecuente entre estos indígenas. Prima el uso de medicamentos farmacéuticos en los Muisca $y$ de plantas medicinales en los Yanakuna. En los Pijao es evidente la complementación terapéutica. Los elementos farmacéuticos se enfocan a un asunto de progreso social y los elementos herbolarios a una cuestión de identidad cultural. En estas comunidades la automedicación en salud bucal es una práctica estructurada y cotidiana en las familias para enfrentar sus dolencias. Esta práctica se soporta en el conocimiento, manejo y disponibilidad de diferentes recursos terapéuticos.

Palabras clave Salud Bucal, Automedicación, Población Indígena, Investigación Cualitativa, Plantas Medicinales 


\section{Introducción}

En Colombia existe un centenar de pueblos indígenas. Esta investigación se interesa en el proceso de automedicación en salud bucal de tres de estos pueblos: Muiscas, Yanakunas y Pijaos. Dicho interés reconoce que la salud de los indígenas latinoamericanos es un tema abordado por entidades sanitarias $^{1-3} \mathrm{y}$ académicas ${ }^{4-6}$. Uno de los aspectos estudiados es su salud bucal, donde se indica la presencia de diversas enfermedades, en particular la caries dental. Igualmente destaca la dificultad en el acceso a los servicios odontológicos, y el uso autónomo y complementario de fármacos y hierbas para solventar las dolencias ${ }^{7}$.

La automedicación es un asunto habitual en estas poblaciones; sin embargo, no se hallaron investigaciones específicas en salud bucal. Los estudios revisados cuantifican el consumo de medicamentos sin prescripción médica y los factores que influyen en esta práctica ${ }^{8-20}$. Igualmente reportan alto uso de fármacos de venta libre como analgésicos y antigripales, y en menor proporción, elementos de venta restringida como los antibióticos. Entre los factores que influyen se indica la escasa educación médica de la población ${ }^{8-11}$, la presencia de malestares de fácil tratamiento ${ }^{8-19}, \mathrm{y}$ de enfermedades crónicas cuidadas durante largo tiempo ${ }^{10,17-19}$. Asimismo se resalta la facilidad para adquirir medicamentos ${ }^{10,13,15,20}$, la influencia de los medios de comunicación en su consumo ${ }^{12,18,20}$, la información adquirida en los servicios sanitarios, incluidas las farmacias ${ }^{10,11,15,16} \mathrm{y}$ las barreras de acceso a los centros de atención a la salud ${ }^{14,16-20}$.

Estas investigaciones consideran que la automedicación es un problema de salud pública debido al consumo "masivo" y "abusivo" de fármacos, y sus efectos negativos en la salud. También insisten en la responsabilidad de la industria farmacéutica y en la falta de control gubernamental. En relación con esta postura, entidades sanitarias nacionales e internacionales promueven estrategias para que el gobierno y la sociedad enfrenten esta problemática, y atiendan sus dolencias de manera científica y segura ${ }^{21-26}$.

Otros estudios revisados utilizaron métodos cualitativos. Éstos indican que la automedicación es una práctica sociocultural ${ }^{27-30}$, y advierten que a partir de la experiencia con sus dolencias, las personas reconocen la eficacia terapéutica de los medicamentos y; por lo tanto, hacen uso de éstos soslayando la atención médica ${ }^{27,28}$, decisión en la que influye su interacción con las farmacias y las barreras de acceso a los servicios de salud ${ }^{20}$. Igualmente se señala el uso de plantas medicina- les porque se conocen sus beneficios curativos, sus propiedades "milagrosas" y sus escasos efectos secudarios ${ }^{27,29,30}$. Ante esta realidad, entidades sanitarias reglamentan la utilización de plantas medicinales, y reconocen que su uso es una práctica terapéutica extendida en la sociedad ${ }^{31,32}$. En general, las investigaciones revisadas consideran a la automedicación como una estrategia de los sujetos para solventar sus dolencias a partir del uso de elementos terapéuticos disponibles, sin la supervisión de profesionales de la salud.

Un autor como Eduardo Menéndez, quien desde la antropología médica crítica examina la interacción entre los elementos simbólicos y estructurales de cada grupo social, considera a la automedicación como la decisión autónoma de los sujetos para utilizar determinados elementos terapéuticos en el tratamiento de sus malestares sin la intervención directa del personal sanitario. Esto no se refiere únicamente a los medicamentos desarrollados por la industria farmacéutica (acetaminofén o antibióticos), sino a todas aquellas sustancias que disponen en su entorno (plantas medicinales, partes de animales, insumos minerales) y que usan las personas y su núcleo social (familia o vecinos) para actuar frente a sus padecimientos $^{33,34}$.

Igualmente indica que la automedicación sintetiza la experiencia de las personas con diferentes modelos de atención a la salud existentes en su territorio (biomedicina, terapias alternativas); por lo tanto, es el principal núcleo de articulación de estos modelos. Dichas experiencias permiten a las personas adquirir conocimientos y habilidades que dinamizan a partir de una racionalidad específica centrada en la eficacia terapéutica, y en sus propias ideas sobre el cuerpo y la salud, y la enfermedad y su atención. Es importante mencionar que la automedicación y el autocuidado hacen parte de la autoatención, forma de atención más frecuente en toda sociedad ${ }^{33,34}$.

La aproximación conceptual de este autor promueve el análisis contextual y específico de cada grupo, sus racionalidades sobre salud y enfermedad, y su relación con los servicios sanitarios. A partir de estos elementos, el objetivo de esta investigación es identificar y analizar el proceso de automedicación en salud bucal en indígenas Muiscas, Pijaos y Yanakunas de Colombia.

\section{Métodos}

Método cualitativo con enfoque etnográfico. Se utilizaron técnicas de investigación como el aná- 
lisis documental, observación participante, diario de campo y entrevista etnográfica. Este método se fundamenta en un acercamiento prolongado y prudente a la vida cotidiana de las comunidades, en donde se observa, pregunta, reflexiona y análiza sobre asuntos específicos, para comprender los sentidos y los significados de las prácticas de las personas ${ }^{35}$; en este caso, lo relacionado a la automedicación en salud bucal.

Estas técnicas se implementaron por parte de tres odontólogos con experiencia etnográfica. El trabajo se desarrolló en tres comunidades: Yanakunas de los Andes del sur del Cauca, Pijaos del valle del alto río Magdalena, y Muiscas de la zona urbana de Bogotá. El análisis documental implicó la búsqueda de la literatura publicada sobre estos pueblos indígenas para conocer sus contextos sociales y asuntos específicos de este estudio. Esta información se constrastó y complementó con la información que emergió a partir de las demás técnicas de investgiación.

La observación participante se desarrolló en varios escenarios con el propósito de conocer la articulación entre las representaciones y las prácticas de los sujetos ${ }^{36}$ respecto a la automedicación en salud bucal; es decir, entre lo que dicen y hacen las personas en este aspecto de su vida. Un escenario fue los hogares de tres familias Muiscas, cinco Yanakunas y seis Pijaos, seleccionadas a partir de criterios como la auto-adscripción étnica, vivir de manera permanente en la región, parentela con uno o más hijos viviendo en casa y su participación voluntaria en el estudio. La elección y el número de familias se soportó en la construcción teórica de tipos ideales ${ }^{34}$, que permite elegir sujetos representativos de cada grupo social.

Esta técnica igualmente se implementó en servicios de salud bucal en las regiones de estudio, $y$ en los lugares en donde se obtienen y/o adquieren los medicamentos. Los primeros se eligieron por la información dada por las familias sobre las entidades sanitarias a las que acuden; y los segundos por ser los entornos de estas comunidades. Esta observación se acompañó del uso del diario de campo, herramienta utilizada de manera permanente para registrar los datos de observaciones y conversaciones, y asimismo, interpretar y reflexionar sobre dicha información ${ }^{35}$.

Las entrevistas se realizaron a personas clave de cada comunidad debido a su trayectoria en el campo de la salud, según el reconocimiento indicado por las familias. Entre los Yanakunas, una yerbatera, un líder comunitario y una promotora de salud; en los Pijao, una mohana o "médica tradicional" y una lideresa comunitaria; y en los Muisca, un líder comunitario, dos adultos mayores y un vendedor de plantas medicinales. Esta técnica permitió obetener información a partir de un diálogo formal orientado por las preguntas de investigación ${ }^{37}$. Las entrevistas fueron grabadas y transcritas.

Toda la información colectada se organizó en un cuadro analítico, técnica que permite ordenar y jerarquizar los datos a partir de categorías establecidas previamente ${ }^{35}$. Asimismo, estos datos se interpretaron desde argumentos teóricos que buscan comprender los sentidos y significados de las prácticas en automedicación, y su relación con elementos estructurales de cada comunidad ${ }^{36}$.

En los aspectos éticos se tuvo en cuenta la Declaración Universal sobre Bioetica y Derechos Humanos de la UNESCO, y la Resolución 8430 de 1993 sobre las normas colombianas de investigación en salud. Asimismo, contó con el aval del Comité de Ética en Investigación de la Facultad de Odontología de la Universidad Nacional de Colombia.

\section{Resultados}

La presentación de resultados inicia con una caracterización de cada comunidad. Los Muiscas de hoy día hablan castellano, y viven en la zona urbana de Bogotá y áreas rurales circunvecinas a 2.600 metros sobre el nivel del mar (m.s.n.m). En esta ciudad habitan principalmente en las localidades de Suba y Bosa (Figura 1), en donde su población es cercana a las 5 mil personas -0.06 por ciento de la población Bogotana ${ }^{38}$-. En general, están integrados a la vida citadina; no obstante, sus condiciones sociales son precarias y residen en lugares segregados donde las instituciones de salud tienen una limitada presencia. Actualmente están en un proceso de reconstrucción política y cultural de su pueblo que incluye el fortalecimiento de su Cabildo Indígena, mismo que es el puente de interacción entre las comunidades y el Estado. Asimismo, promueven el rescate y defensa de elementos materiales e inmateriales de su cultura, entre los cuales destaca su "medicina ancestral" y sus conocimientos sobre plantas de uso terapéutico, sin que ello implique un desinterés en la medicina occidental.

Los Yanakuna habitan los Andes al sur del país, en el llamado Macizo Colombiano, entre los 2.500 y 3.300 m.s.n.m. (Figura 1). Su población es cercana a los 45 mil habitantes -0.09 por ciento de la población colombiana-, y su principal acti- 


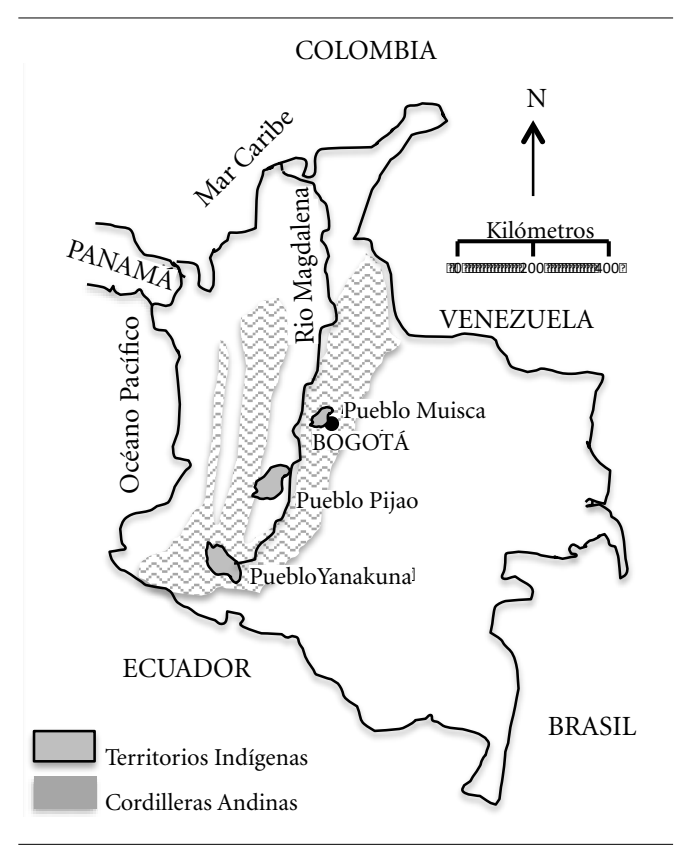

Figura 1. Territorio de los Pueblos Muisca, Yanakuna y Pijao en Colombia.

Fuente: Desarrollada por los autores.

vidad económica es la agricultura en tierras colectivas denominadas resguardos ${ }^{39}$. Aunque este pueblo está conformado por 31 comunidades, solo se interactuó con gente de Kakiona y Pancitará, territorios donde conviven cerca de cuatro mil individuos. Este grupo indígena es de origen Quechua; no obstante, solo hablan castellano. Las comunidades se organizan en el Cabildo Mayor Yanakuna, entidad política que promueve un Plan de Vida en donde destaca su modelo intercultural de salud y una institución denominada "Runa Yanakuna" que presta servicios de atención médica occidental y tradicional.

Los Pijao ocupan el cálido valle del río Magdalena, entre los 500 y 1.000 m.s.n.m., al sur departamento del Tolima (Figura 1). Su idioma desapareció a mediados del siglo XX y su población se acerca a los 60 mil habitantes -4 por ciento de la población indígena naciona ${ }^{40}$. Viven en un centenar de comunidades en tierras colectivas en donde cultivan platano, yuca, maíz, fríjol, café y caña de azucar, y crían ganado vacuno a pequeña escala. En este caso se interactuó con las comunidades Anabá y El Vergel-Calarma, municipio de Ortega, donde habitan 300 personas. Se han organizado para recuperar sus territorios ancestrales y fortalecer su autonomía territorial en dimensiones como la educación y la salud. En el tema sanitario existe un proceso de atención intercultural en salud que es desarrollado por una institución denominada The Wala.

En relación a la atención sanitaria, es importante subrayar que la población indígena de Colombia tiene un seguro en salud subsidiado por el Estado; aun así, en todo el país los servicios odontológicos mantienen diferentes barreras de acceso para esta población. Éste es uno de los motivos por los cuales estas comunidades están implementando instituciones de salud con carácter autónomo e intercultural.

El trabajo etnográfico permitió identificar que las estrategias de automedicación en salud bucal están contextualizadas y se relacionan con cuatro aspectos: las representaciones que los sujetos tienen sobre la boca; el impacto de los padecimientos bucales sobre la vida cotidiana de las personas; la interacción de las familias con las diferentes formas de atención a la salud existentes en sus territorios; y la disponibilidad de elementos de utilidad terapéutica.

Sobre el primer aspecto, entre los Muisca la boca tiene funciones relacionadas con el bienestar integral: comer y comunicar, así como expresar mediante la risa, amar a través de los besos o presumir con diseños de sonrisa. Para los Yanakunas la boca es una herramienta con múltiples partes, útil para comunicarse y alimentarse, soporte fundamental en la vida cotidiana, donde el trabajo en grupo es fundamental. Igualmente, en su cavidad bucal se representan explicaciones mágicas sobre de ciertas enfermedades; por ejemplo, sobre las aftas bucales se indica que aparecen por la interacción de las personas con un insecto bioluminicente denominado ninacuro, larva endémica de esta zona andina. Entre los Pijao, la boca es parte fundamental del cuerpo, elemento para la comunicación social y la masticación de los alimentos; por este motivo la lengua, los labios y los dientes son componentes primordiales que deben ser cuidados con esmero porque pueden ser atacados por diversos padecimientos.

El segundo asunto refiere que para los Yanakuna y los Pijao el dolor de origen dental generalmente incapacita a la gente para el desarrollo de las actividades cotidianas; motivo para buscar soluciones inmediatas y eficaces. Los Muiscas consideran que además de la odontalgia, la inflamación y el sangrado de las encías son situaciones desfavorables, debido que impactan en la apariencia estética e indican el nivel de cuidado de la salud bucal.

Respecto al tercer elemento, los Muiscas tienen una amplia oferta de servicios a los cuales 
asisten pese a las barreras de acceso, y ocasionalmente asumen los costos de su atención en consultorios privados. En ocasiones esta población interacciona con los "abuelos ancestrales" en busca de soluciones a sus dolencias. Los servicios de atención odontológica para los Yanakunas son insuficientes y distantes y, asimismo, asisten donde las yerbateras. Los Pijao también tienen barreras de acceso a los servicios de salud, y además visitan a un odontólogo empírico debido a su bajo costo y fácil acceso, y a la Mohana, cabeza médica de su medicina tradicional.

En relación al último asunto, la información se organiza en el Cuadro 1 que presenta el tipo de malestar, los recursos terapéuticos utilizados, el lugar de obtención y su forma de preparación, así como el pueblo indígena que hace uso de estos recursos como elemento curativo. Los Muiscas disponen en su zona de farmacias en donde adquieren medicamentos de origen biomédico. Igualmente, obtienen plantas medicinales en los mercados, y de manera particular en el Cusmuy, casa ceremonial construida en la ciudad donde cultivan hierbas terapéuticas. Los Yanakunas consiguen analgésicos en tiendas veredales, y cosechan plantas medicinales en montes y huertas. En la región Pijao es fácil obtener medicamentos para los dolores dentales; malestares que también se atienden con yerbas curativas recolectadas en jardines, potreros y plantíos (Cuadro 1).

Otros datos etnográficos indican con detalle la manera en que estas comunidades materializan sus estrategias de automedicación en salud bucal. En esta labor se identificó que la automedicación se dinamiza una vez aparece un malestar, que es liderada por las madres y mujeres de mayor edad, y que se hace uso de diferentes elementos y técnicas terapéuticas.

$\mathrm{Al}$ aparecer una molestia en la boca, las cuidadoras utilizan su experiencia para reconocer su impacto en la salud y en la vida cotidiana del afectado $y$, en consecuencia, orientan una respuesta inmediata en el escenario doméstico. Este contacto entre la cuidadora y el paciente incluye un diagnóstico sobre la intensidad, persistencia y afectación de dicha molestia, y sobre estas evidencias activan sus saberes sobre la enfermedad y su atención, argumento que incluye revisar la disponibilidad de recursos terapéuticos en el entorno del hogar.

En cada grupo indígena estudiado este asunto es diferente. Entre los Muiscas dichos saberes se complementan con el apoyo del buscador Google y el fácil acceso a las farmacias. Entre Yanakunas y Pijaos son importantes los conocimientos y habilidades de los vecinos y la disponibilidad de plantas de uso medicinal en el entorno. Asimismo, es habitual que las familias Muiscas tengan en sus viviendas fármacos de uso común, sobre todo contra el dolor, la inflamación y la fiebre, situación poco frecuente entre Yanakunas y Pijaos.

El malestar más habitual en la boca es el dolor, y en específico, la odontalgia. Para enfrentarlo, en las tres comunidades se utilizan elementos de origen farmacéutico. Acetaminofén o Paracetamol, medicamentos de fácil adquisición. Si el dolor persiste o es "fuerte", las familias recurren a las farmacias para comprar el Ibuprofeno, incluso reconocen que existen diferentes dosis y frecuencias de consumo de este fármaco. Además de estos medicamentos, los Muiscas emplean Diclofenaco, Naproxeno y Ponstan -ácido mefenámico-. Frente a la inflamación, en las tres comunidades usan acetaminofén o paracetamol, y ante las infecciones, los Muiscas y Pijaos recurren a la Amoxicilina, y los Yanakunas a la Ampicilina, antibióticos que se obtienen fácilmente en las farmacias aunque su venta requiere receta médica.

Esta población no conoce los componentes ni los mecanismos de acción de estos fármacos. Su uso se origina y fortalece por experiencias con resultados eficaces, y socializadas en el ámbito familiar y comunitario. Dicha eficacia terapéutica no es de conocimiento exclusivo de las cuidadoras; cada individuo elabora un razonamiento específico sobre estos medicamentos a partir de sus propios aprendizajes en el manejo de sus dolencias. Igualmente, se halló que dichos medicamentos no son exclusivos para el dolor en la cavidad bucal; son utilizados para enfrentar toda clase de dolor, diferenciando su utilización de acuerdo con la intensidad y duración.

Otro elemento utilizado entre los Muiscas y los Pijaos contra estos dolores es el linimento negro-mezcla de extractos y aceites-, medicamento líquido sin registro sanitario que se obtiene en tiendas de productos naturales y algunas farmacias, y que se impregna en una mota algodón y se coloca en la cavidad cariada. Este procedimiento también se realiza con las llamadas "calzas eléctricas" -solución con benzocaína y antipirina-, y otros elementos como clavos de olor, alcohol antiséptico, esmalte para uñas y betún para los zapatos. Hay insumos como la piedra lumbre y la sal de vigua, los cuales se diluyen en agua y se utilizan en forma de colutorios para hacer frente al dolor leve, y para inflamaciones, infecciones, aftas y hemorragias.

Las plantas de uso medicinal son otros recursos terapéuticos. De éstas se usan hojas, tallos, la- 
Cuadro 1. Elementos terapéuticos utilizados en la autoatención en salud bucal por la población Muisca, Yanakuna y Pijao.

\begin{tabular}{|c|c|c|c|c|}
\hline \multirow{2}{*}{ Malestar } & \multicolumn{3}{|c|}{ Estrategia de Automedicación } & \multirow{2}{*}{$\begin{array}{c}\text { Pueblo } \\
\text { Indígena }\end{array}$} \\
\hline & Elemento Terapéutico & Fuente del Recurso & Método de Uso & \\
\hline Dolor & $\begin{array}{l}\text { Paracetamol, } \\
\text { Ibuprofeno, } \\
\text { Acetaminofén }\end{array}$ & $\begin{array}{l}\text { Tienda veredal y barrial } \\
\text { Farmacia }\end{array}$ & Tabletas & $\begin{array}{l}\text { Yanakuna, } \\
\text { Muisca, Pijao }\end{array}$ \\
\hline Dolor & $\begin{array}{l}\text { Diclofenaco, Naproxeno, } \\
\text { Ponstan }\end{array}$ & Farmacia & Tabletas & Muisca \\
\hline Dolor & $\begin{array}{l}\text { Linimento negro, calza } \\
\text { eléctrica }\end{array}$ & $\begin{array}{l}\text { Tienda naturista y } \\
\text { farmacia }\end{array}$ & $\begin{array}{l}\text { En mota de algodón en } \\
\text { cavidad cariada }\end{array}$ & Muisca, Pijao \\
\hline Dolor & $\begin{array}{l}\text { Alcohol antiséptico, } \\
\text { alcohol etílico }\end{array}$ & $\begin{array}{l}\text { Tienda veredal y barrial } \\
\text { Farmacia }\end{array}$ & $\begin{array}{l}\text { En mota de algodón en } \\
\text { cavidad cariada }\end{array}$ & $\begin{array}{l}\text { Yanakuna, } \\
\text { Muisca, Pijao }\end{array}$ \\
\hline Dolor & $\begin{array}{l}\text { Esmalte para uñas, } \\
\text { betún para zapatos }\end{array}$ & Tienda veredal y barrial & $\begin{array}{l}\text { En mota de algodón en } \\
\text { cavidad cariada }\end{array}$ & $\begin{array}{l}\text { Pijao, Muisca, } \\
\text { Yanakuna }\end{array}$ \\
\hline Dolor & $\begin{array}{l}\text { Yerbabuena, Chilcarusia } \\
\text { Coca, Durazno }\end{array}$ & Huerta, potreros, bosques & Cocción hojas y colutorio & Yanakuna \\
\hline Dolor & Lechero, amapola & Huerta, potreros, bosques & Latex en cavidad cariada & Yanakuna \\
\hline Dolor & $\begin{array}{l}\text { Dormidera. Coca, } \\
\text { Marihuana }\end{array}$ & Huerta, potreros, bosques & $\begin{array}{l}\text { Cocción hojas y tallo } \\
\text { Colutorio }\end{array}$ & Pijao \\
\hline Dolor & Clavo de olor, Ajo & Tienda veredal y barrial & $\begin{array}{l}\text { Macerado y puesto en la } \\
\text { cavidad cariada }\end{array}$ & Muisca, Pijao \\
\hline Dolor & Romero & $\begin{array}{l}\text { Jardines, ventas } \\
\text { ambulantes }\end{array}$ & $\begin{array}{l}\text { Cocción de hojas } \\
\text { Colutorio }\end{array}$ & Muisca \\
\hline $\begin{array}{l}\text { Dolor, } \\
\text { infección }\end{array}$ & $\begin{array}{l}\text { Sal de Vigua, piedra } \\
\text { lumbre }\end{array}$ & $\begin{array}{l}\text { Farmacias, tienda veredal } \\
\text { y barrial }\end{array}$ & $\begin{array}{l}\text { Solución en agua } \\
\text { Colutorio }\end{array}$ & $\begin{array}{l}\text { Pijao, Muisca, } \\
\text { Yanakuna }\end{array}$ \\
\hline Infección & Amoxicilina, Ampicilina & Farmacia & Cápsulas & $\begin{array}{l}\text { Pijao, Muisca, } \\
\text { Yanakuna }\end{array}$ \\
\hline Infección & Morochillo & Bosques & $\begin{array}{l}\text { Punción con astilla en } \\
\text { absceso }\end{array}$ & Yanakuna \\
\hline Infección & Uchuva & $\begin{array}{l}\text { Huertas, potreros, } \\
\text { bosques }\end{array}$ & $\begin{array}{l}\text { Cocción de hojas y frutos } \\
\text { Colutorio }\end{array}$ & Yanakuna \\
\hline Infección & $\begin{array}{l}\text { Llanten, Chipaca, } \\
\text { Arrayán }\end{array}$ & $\begin{array}{l}\text { Huertas, potreros, } \\
\text { bosques }\end{array}$ & $\begin{array}{l}\text { Cocción de hojas } \\
\text { Colutorio }\end{array}$ & Pijao \\
\hline Infección & Tabaco & Tiendas barriales & $\begin{array}{l}\text { Emplasto en el área } \\
\text { infectada }\end{array}$ & Muisca \\
\hline Inflamación & $\begin{array}{l}\text { Malva, Sábila, } \\
\text { Yerbamora, Cáncer }\end{array}$ & Huerta, potreros, bosques & $\begin{array}{l}\text { Cocción de hojas } \\
\text { Emplasto }\end{array}$ & Yanakuna \\
\hline Inflamación & Caléndula & Huertas, Jardines & $\begin{array}{l}\text { Cocción de hojas y flores } \\
\text { Colutorio }\end{array}$ & Muisca, Pijao \\
\hline Hemorragias & Salvia, Geranio Blanco & Huertas & $\begin{array}{l}\text { Cocción de hojas } \\
\text { Emplasto }\end{array}$ & Yanakuna \\
\hline
\end{tabular}

Fuente: Desarrollada por los autores a partir de datos etnográficos.

tex o cortezas en forma de colutorios, emplastos o infusiones, y se obtienen en huertas, potreros, jardines y bosques, y también en los mercados. Varias de éstas son de origen Andino -Uvilla, Arrayán-, de zonas tropicales -Dormidera, Cocay otras provienen de latitudes como Arabia -Sábila- o el Mediterráneo -Caléndula. Es importante subrayar que cada comunidad vive en un espacio geográfico determinado; por lo tanto, su flora medicinal es específica.

Para los Yanakunas las plantas son el principal elemento terapéutico. Frente al dolor usan hojas de Yerbabuena -Menta spicata-, Chilcarusia -Stelvia Lucida-, Coca -Erythroxilon cocca-, o Durazno -Durus acinus-; también latex de Lechero -Euphorbia lactiflua-, o Amapola -Papaver 
somniferum. Para desinflamar follaje de Malva -Malacara alceifolia-, Salvia -Salvia officinalis-, o Yerbamora -Solanum nigrum-; y para las hemorragias cataplasmas de geranio blanco -Pelargonium. Los abscesos son drenados mediante una punción con una astilla de morochillo -Miconia theazana-, y la aftas e inflamación de la garganta son tratadas con el zumo de uvilla -Phisalis peruvicona.

Los Pijao usan plantas con menor frecuencia, y disponen de éstas como medida inicial ante sus dolencias, pero si el malestar continúa o es fuerte acuden a los fármacos por su eficacia. Para el dolor utilizan clavos de olor-Syzygium aromaticum-, infusiones de tallos de Dormidera -Mimosa púdica-, o hojas de Marihuana -Cannabis sativa- y Coca. Para la inflamación Caléndula -Caléndula officinalis-, y como desinfectante Llantén -Plantago major-, Chipaca-Bidens pilosa-, o Arrayán -Myrtus communis.

La vida urbana Muisca no impide la utilización de plantas medicinales. Para la odontalgia, clavos de olor, o pasta de ajo -Allium sativum- o de arrayán que se coloca en cavidad cariada. El Romero -Rosmarinus officinalis- para aliviar los dolores posoperatorios, el tabaco -Nicotiana tabacum- contra las infecciones, y Yerbamora y Caléndula como desinflamatorio.

Es fundamental indicar que, en ocasiones, la eficacia de las plantas medicinales se complementa con fármacos, dependiendo de la intensidad y la duración del malestar. Entre los Yanakunas el ibuprofeno se consume con agua de Yerbabuena, y entre los Pijaos con infusión de Dormidera.

\section{Discusión}

Los resultados permiten discutir varios elementos del proceso de automedicación. Uno de éstos es la relación entre territorio y salud, y las representaciones cotidianas de la población respecto a la vida, la salud, la enfermedad y la muerte. El otro elemento es la interacción de los sujetos con los sistemas de salud existentes en sus territorios, asunto que incluye la apropiación, disponibilidad y acceso a recursos terapéuticos. A partir de estos elementos es posible plantear una tensión con las nociones existentes sobre automedicación -en salud bucal.

En detalle, se encontró que cada comunidad habita un territorio donde prevalece una representación equilibrada sobre la boca, sus malestares y su atención. Así, el territorio es un corpus heurístico que entrama aspectos estructurales y simbólicos del campo de la salud ${ }^{41}$, y en él coexisten diferentes sistemas de atención a la salud ${ }^{42} \mathrm{y}$ sus múltiples recursos materiales e inmateriales utilizados para dar respuesta a los inconvenientes en salud ${ }^{43}$.

En la boca se representa el sufrimiento (dolor), el deseo (beso) o el pecado (caries), e igualmente símbolos corporales de estatus social (diseño de sonrisa). La automedicación se ocupa del sufrimiento, estrategia inmediata para remediar el pecado. La atención odontológica también se encarga de esta tarea, pero su labor es exclusiva en lo relacionado al estatus social. La boca es entonces un espacio de intervención terapéutica diferenciada, evidencia de la desigualdad social ${ }^{44}$ e igualmente un escenario de funciones que establecen una compleja diversidad de prácticas y relaciones en una u otra comunidad ${ }^{45}$.

Las representaciones sobre la boca se relacionan con explicaciones sobre sus enfermedades. En los Yanakuna los razonamientos sobre el origen de algunos padecimientos provienen de la medicina tradicional. En los Pijao las explicaciones evidencian una articulación entre la biomedicina y la medicina tradicional, y en los Muisca es notable el peso de los argumentos biomédicos. Esto indica que cada comunidad interactúa en sus territorios con otros grupos sociales, y tiene una integración desigual a la sociedad nacional, integración subordinada en donde la biomedicina es uno de los instrumentos de poder y control $^{42}$.

Diversos investigadores enfatizan que las lógicas utilizadas para entender la enfermedad se corresponden con los raciocinios para su atención ${ }^{46-48}$. No obstante, cada sujeto tiene experiencias particulares con sus malestares bucales, particularmente con el dolor dental que en ocasiones se convierten en tormento y exige atención inmediata que demanda diversidad de técnicas y medicamentos. En este proceso se introducen nuevos insumos para superar estas dolencias a través del método de ensayo y error, y cuyos resultados positivos son socializados por los sujetos en su entorno social inmediato.

La automedicación es un proceso dirigido por madres y abuelas a partir de conocimientos y habilidades transmitidas generacionalmente, y por el contacto con los servicios de salud presentes en sus territorios ${ }^{27-30,33,34,42-44,46-48}$. Por este motivo, en los Muisca de Bogotá priman procedimientos y medicamentos que se corresponden con lógicas biomédicas, y en los Yanakuna andinos prevalecen tratamientos e insumos terapéuticos que provienen de la medicina tradicional. 
En la automedicación se apropia una amplia gama de elementos curativos. Se reconoce su capacidad medicinal, técnicas de utilización, manera de obtenerlos y algunos efectos adversos. Los elementos de mayor uso son los medicamentos farmacéuticos debido a su fácil adquisición y eficacia terapéutica ${ }^{8-23,27-30}$, cuestión que para diferentes autores se debe a la falta de educación médica de la población ${ }^{8-11}$. No obstante, se subraya que la educación en salud se debe centrar en acciones de promoción y prevención, y no exclusivamente en la atención ${ }^{49}$, y que en el tema de medicamentos la gente es esencialmente educada por los servicios de salud y la industria farmacéutica. Allí donde no hay servicios de salud, el consumo de medicamentos -con o sin receta médica- es inferior; por lo tanto, la mala educación médica por parte de las instituciones sanitarias y las barreras de acceso a los servicios de atención a la salud ${ }^{14,16-20}$, motivan la automedicación.

Otros elementos ampliamente utilizados son las plantas ${ }^{27,29,30,42-44,46-48}$. En los grupos indígenas éstas son una opción primordial para enfrentar los malestares bucales debido a que se reconocen sus propiedades curativas ${ }^{2,4}$. Su uso también tiene un soporte ideológico por la idea de una naturaleza benigna $^{27,29,30}$, y un sustento político que esgrime los recursos curativos del territorio. Las plantas también son usadas como complemento a medicamentos farmacéuticos ${ }^{42-44,46-48}$; así se reconoce la eficacia de estos medicamentos y que los recursos vegetales representan una parte subjetiva del cuidado y funcionan como un regulador de los efectos secundarios de los productos farmacéuticos.

En general, la automedicación en salud bucal en estas comunidades es un proceso sociocultural que implementan los sujetos de manera autónoma sin la intervención directa de personal sanitario $^{27-30,33,34,42-44,46-48}$. Este proceso pretende restablecer la salud o limitar los efectos de la enfermedad mediante la utilización de diversos elementos y técnicas terapéuticas disponibles en el entorno, utilizadas a partir de razonamientos determinados que tiene cada colectivo sobre el cuerpo y la salud, y la enfermedad y su atención. Dichos razonamientos son específicos, y en éstos se articulan conocimientos y habilidades que provienen de los diferentes sistemas de atención a la salud con los cuales interactúa cada una de estas comunidades.

El centro de este proceso son las personas y la capacidad que tiene cada grupo social para entender y atender de manera autónoma sus enfermedades. Por lo tanto, contrasta con conceptos que consideran a la automedicación como una contravención en el consumo de medicamentos de origen farmacéutico ${ }^{8-20,50,51}$, y que se centran en este tipo de recurso terapéutico como una representación científica de los servicios sanitarios. Y si bien se reconoce que el uso "masivo" y "abusivo" de todo tipo de medicamentos es un problema para la salud de la población ${ }^{8-20}$, cabe señalar que la automedicación es un método para comprender el nivel de desarrollo de los sistemas de atención a la salud.

\section{Conclusiones}

La automedicación en salud bucal en indígenas Muiscas, Pijaos y Yanakunas de Colombia es un proceso que relaciona recursivamente diferentes dimensiones sociales. Una de estas dimensiones es el territorio, espacio simbolizado donde cada comunidad construye y dinamiza sus representaciones sobre la boca y sus componentes, el bienestar y la salud, y las enfermedades bucales y la atención a las mismas. Igualmente es un escenario de vida cotidiana donde las comunidades promueven su autodeterminación política; y uno de los resultados de este ejercicio de poder es la construcción de sistemas sanitarios con una perspectiva intercultural.

Otro escenario social se dinamiza a partir del pluralismo médico; es decir, se reconocen y utilizan varios sistemas de atención a la salud. En la automedicación se articulan recursos terapéuticos provienentes de diversos sistemas médicos para hacer frente a un mismo malestar bucal. Por este motivo, se evidencian asimetrías en la interacción complementaria entre medicamentos farmacéuticos y medicamentos vegetales. Esto indica el nivel de integración y conflicto que tienen la biomedicina y la medicina tradicional en la vida sociocultural de cada colectivo.

Una más de estas dimensiones reflexiona desde la perspectiva antropológica de este estudio, y que considera que todo recurso terapéutico utilizado de manera intensional es considerado un medicamento. Al respecto, cada comunidad entiende y atiende de manera específica sus enfermedades, y como corolario, existen racionalidades específicas sobre los medicamentos. Éstas incluyen el lugar y manera de adquirirse y utilizarse, la dosis a emplear y su complementariedad con otros medicamentos, y la eficacia terapéutica que corresponde con experiencias individuales del contexto microsocial.

La última dimensión para señalar es la apropiación del cuerpo y sus dolencias. En el proceso 
de automedicación los sujetos ejercen el control en su territorio primigenio: su cuerpo y en particular su boca. Sus dolencias y su conciencia lo llevan a movilizar sus conocimientos y habilidades para restaurar su salud de manera inmediata $e$ independiente de las dinámicas que priman en los sistemas de salud (medicina occidental y tradicional), sistemas con las cuales tienen posiblidades de interactuar y que en general están mediados por transacciones de dinero, tiempo y dignidad.

\section{Colaboradores}

EJO Lozano construyó el proyecto de investigación y gestionó el apoyo económico para su realización. Asimismo, orientó y participó en el trabajo etnográfico en las tres comunidades donde se desarrolló el proyecto, y organizó, analizó y escribió el texto final con los aportes de los otros dos investigadores. YDL Pinzón apoyó en la construcción del proyecto de investigación y en la gestión de recursos económicos. Igualmente participó en el trabajo etnográfico, en la organización y análisis de la información, y en la escritura del texto final. SIP Solano participó en la construcción del proyecto de investigación, en el trabajo etnográfico, en la organización y análisis de la información, y en la escritura del texto final.

\section{Referencias}

1. Organización Panamericana de la Salud (OPS). Una visión de salud intercultural para los pueblos indios de las Américas. Washington: OPS; 2008.

2. Organización Panamericana de la Salud (OPS). La Salud de los Pueblos Indígenas de las Américas: Conceptos, Estrategias, Prácticas y Desafíos. Quito: OPS; 2009.

3. Ministerio de Salud y Protección Social (MSP). Organización Panamericana de la Salud (OPS). Insumos para la conceptualización y discusión de una politica de protección social en salud para los grupos Étnicos de Colombia. Bogotá: Nuevas Ediciones Limitada; 2004.

4. Montenegro R, Stephens C. Indigenous health in Latin America and the Caribbean. Lancet 2006; 367(9525): 1859-1869.

5. Menéndez E. Antropología médica en América Latina 1990-2015: Una revisión estrictamente provisional. Salud Colect 2018; 14(3):461-481.

6. Langdon E, Cardoso MD. Saude indígena: políticas comparadas na America Latina. Florianópolis: Editora da UFSC; 2015.

7. Albarrán-Paredes Y, Berbesí-Jerez Y, Hernández-Rondón I, Marín-Altuve E, Rodríguez-González M, Uzcátegui-Montes M, Velasco-Quintero É. Salud bucal en indígenas latinoamericanos. Una revisión sistemática. Rev Venez Investig Odontol 2017; 5(2):238-262.

8. González-Amayuelas M, Mendibil-Crespo I, Gutiérrez-Martínez I, Blanco-Alonso M. Automedicación con AINE por los usuarios de dos consultas de Atención Primaria. Gac Med Bilbao 2006; 103(2):54-57.

9. Reynoso J, Mejía B, Olvera H, Chehue R. Prevalencia de la automedicación y del consumo de remedios herbolarios entre los usuarios de un centro de salud. Rev Mex Cien Farm 2008; 37(4):30-34.

10. Rodilla E, Dagá I, Giralt M, Algué A, Comalada M, Corbera M. Automedicación y ancianos. La realidad de un botiquín casero. Aten Primaria 2009; 41(5):269274.

11. Suleman S, Ketsela A, Mekonnen Z. Assessment of self-medication practices in Assendabo town, Jimma zone, southwestern Ethiopia. Res Social Adm Pharm 2009; 5(1):76-81.

12. Vitor R, Lopes C, Menezes H, Kerkhoff C. Padrão de consumo de medicamentos sem prescrição médica na cidade de Porto Alegre. Cien Saude Colet 2008; 13(Supl.):737-743.

13. Marulanda F. Estudio sobre automedicación en la Universidad de Antioquia, Medellín, Colombia. Iatreia 2002; 15(4):242-247. 
14. López J, Dennis R, Moscoso S. Estudio sobre la automedicación en una localidad de Bogotá. Rev Salud Publica 2009; 11(3):432-442.

15. Villegas F, Nasner K, Gómez D, Cruz S, Ruden S, Bedoya J, Gómez R. Patrón de automedicación en la zona urbana de la ciudad de Pereira (Colombia) en el trimestre marzo-mayo 2013. Investigaciones Andina 2014; 16(29):1073-1085.

16. Yousef A, Al-Bakri A, Bustanji Y, Wazaify M. Self-medication patterns in Amman, Jordan. Pharm World Sci 2008; 30(1):24-30.

17. Figueiras A, Caamano F, Gestal-Otero J. Sociodemographic factors related to self-medication in Spain. Eur J Epidemiol 2000; 16(1):19-26.

18. Tobón F, Montoya S, Orrego M. Automedicación familiar, un problema de salud pública. Educ Med 2018; 19:122-127.

19. Altagracia M, Kravzov J, Moreno S, Ríos C, Vázquez $M$. Self-medication in rural and urban communities in the state of Guerrero Mexico. Rev Mex Cien Farma 2003; 34:27-35.

20. Cesolari J, Calvi B, Garrote N, Pérez B, Busmail L. Automedicación, un problema de educación médica. Rev Med Rosario 2004; 70(1):140-142.

21. World Health Organization (WHO). Guidelines for the Regulatory Assessment of Medicinal Productos for Use un Self-medication. Ginebra: WHO; 2000.

22. World Health Organization (WHO). The Benefits and Risks of Self-medication. Ginebra: WHO; 2000.

23. Organización Mundial de la Salud (OMS). El rol de los farmacéuticos en el auto-cuidado y la automedicación. La Haya: OMS; 1998.

24. Organización Panamericana de la Salud (OPS). Legislación sobre antibióticos en América Latina. Washington: OPS; 2004.

25. Ministerio de Salud y Protección Social (MSP). Política Farmacéutica Nacional de Colombia y sus Desarrollos Normativos. "Presentación de experiencias y buenas prácticas en cuanto a medida regulatorias de medicamentos en Colombia”. Bogotá: MSP; 2009.

26. Ministerio de Salud y Protección Social (MSP). MinSalud promueve los 12 mandamientos contra la resistencia antimicrobiana. Bogotá: MSP; 2017.

27. Juárez E. La autoatención en un grupo de ancianos con diabetes residentes en Iztapalapa DF. Cuicuilco 2005; 12(33):9-26.

28. Loyola A, Lima-Costa M, Uchôa E. Bambuí. Project: a qualitative approach to self-medication. Cad Saude Publica 2004; 20:1661-1669.

29. Pilar M, Morales T, Valdés A, Sorí E, Luis M. Automedicación ¿beneficio o perjuicio? Gac Med Espirituana 2017; 5(3):1-10.

30. Rubio M, Pérez A, Puerta Z, Avila I. Automedicación y creencias en torno a su práctica en Cartagena, Colombia. Rev Cuidarte 2017; 8(1):1509-1518.

31. Organización Mundial de la Salud (OMS) Estrategia de la OMS sobre medicina tradicional. 2014-2023. Hong Kong: OMS; 2014.

32. Mundial de la Salud (OMS). Situación reglamentaria de los medicamentos. Una reseña mundial. Ginebra: OMS; 2000.

33. Menéndez E. Modelos de atención de los padecimientos: de exclusiones teóricas y articulaciones prácticas. Cien Saude Colet 2003; 8(1):185-207.
34. Menéndez E. Modelo hegemónico, modelo alternativo subordinado, modelo de autoatención, caracteres estructurales. En: Campos R. La antropología médica en México, Tomo I. México D.F.: Editorial Antologías Universitarias; 1992. p. 97-114.

35. Restrepo E. Etnografía: alcances, técnicas y éticas. Bogotá: Envión Editores; 2016.

36. Menéndez E. Técnicas cualitativas, problemátización de la realidad y mercado de saberes. Cuad Antropol Soc 2001; 13:9-51.

37. Guber R. La etnografía. Método, campo, reflexividad. Buenos Aires: Editorial Norma; 2001.

38. República de Colombia. Ministerio del Interior (MI). Pueblo Muisca. Bogotá: MI; 2019.

39. República de Colombia. Ministerio del Interior (MI). Pueblo Yanacona. Bogotá: MI; 2019.

40. República de Colombia. Ministerio del Interior (MI) Pueblo Pijao Coyaima-Natagaima. Bogotá: MI; 2019.

41. Barabas AM. Etnoterritorios y rituales terapéuticos en Oaxaca. Scripta Ethnol 2002; 24:9-19.

42. Menéndez, E. El modelo médico hegemónico: transacciones y alternativas hacia una fundamentación teórica del modelo de autoatención en salud. Arxiu D’etnografía Catalunya 2016; 3:84-119.

43. Hersch-Martínez P. Epidemiología sociocultural: una perspectiva necesaria. Salud Publica Mex 2013; 55(5):512-518.

44. Ospina E, Bellamy C. Autoatención en salud oral en el pueblo Yanacona de los Andes del sur de Colombia. Acta Odontol Colombiana 2011; 1(2):85-101.

45. Malagón R. La boca como representación. Segunda parte: Los límites y las negociaciones. En: Viveros M, Garay A. Cuerpos, diferencias y desigualdades. Santa Fe de Bogotá: Universidad Nacional de Colombia; 1999. p. 95-109.

46. Menéndez E. Salud intercultural: propuestas, acciones y fracasos. Cien Saude Colet 2016; 21(1):109-118.

47. Mendoza Z. Enfermedad ¿para quién?”. Rev Nueva Antropol 1997; 16(52):117-139.

48. Osorio R. Entender y atender la enfermedad, los saberes maternos frente a los padecimientos infantiles. Biblioteca de la medicina tradicional mexicana. México D.F.: Conaculta; 2001.

49. Acevedo M, Becerra F, Ospina J, Paucar G, Córdoba A Correa F. El diálogo de saberes como posición humana frente al otro: referente ontológico y pedagógico en la educación para la salud. Investig Educ Enferm 2009; 27(1):104-111.

50. Kregar G, Filinger E. ¿Qué se entiende por automedicación? Acta Farm Bonaerense 2005; 24(1):130-133.

51. Wirtz V, Dreser A, Leyva R. El debate sobre la automedicación. Salud Publica Mex 2009; 51:179-180.

Artículo presentado en 18/03/2019

Aprobado en 07/02/2020

Versión final presentada en 09/02/2020

Editores jefes: Romeu Gomes, Antônio Augusto Moura da Silva 